CERN-TH/97-129

\title{
Supersymmetry Breaking with Vanishing F-terms in Supergravity Theories
}

\author{
Gia Dvali $^{(1)}$ and Alex Pomarol ${ }^{(2)}$ \\ ${ }^{(1)}$ Theory Division, CERN \\ CH-1211 Geneva 23, Switzerland \\ (2) Institut de Física d'Altes Energies \\ Universitat Autònoma de Barcelona \\ E-08193 Bellaterra, Barcelona, Spain
}

\begin{abstract}
In conventional supergravity theories, supersymmetry is broken by a non-zero $F$-term, and the cosmological constant is fine tuned to zero by a constant in the superpotential $W$. We discuss a class of supergravity theories with vanishing $F$-terms but $\langle W\rangle \neq 0$ being generated dynamically. The cosmological constant is assumed to be cancelled by a non-zero $D$-term. In this scenario the gravity-mediated soft masses depend only on a single parameter, the gravitino mass. They are automatically universal, independently of the Kähler metric, and real. Thus, dangerous flavor or CP violating interactions are suppressed. Unlike in conventional supergravity models, the Polonyi problem does not arise.
\end{abstract}

CERN-TH/97-129

June 1997 


\section{Introduction}

One of the major challenges in supersymmetric models for particle physics is the understanding of the breaking of supersymmetry. Supergravity theories offer one of the simplest scenarios [1]. Supersymmetry is broken by an F-term of a hidden superfield, and gravity plays the role of transmitting the supersymmetry breaking to the ordinary quarks and leptons. Nevertheless, the induced soft breaking masses are not generically universal [2] nor real [3], leading to dangerous flavor and $\mathrm{CP}$ violating interactions.

Here we will consider a different scenario of supergravity breaking. The $F$-terms of the hidden sector superfields will be zero, but not their superpotential. This will be generated dynamically at a low-energy scale. By setting the cosmological constant to zero (with a nonzero $D$-term), the induced superpotential will parametrize the breaking of supersymmetry. Like in conventional supergravity models, gravity will mediate the breaking of supersymmetry to the ordinary quarks and leptons. Nevertheless, in our scenario the scalar masses turn to be automatically universal, independently of the Kähler metric. The origin of universality is a consequence of the super-Weyl-Kähler symmetry of supergravity. We find that the trilinears and gaugino masses are zero at tree-level. Nevertheless, gaugino masses are generated at the one-loop level if heavy states are present in the theory. This is the case of grand unify theories (GUTs) where the large number of heavy states can induce sizeable gaugino masses. We will show that our scenario do not suffer from the supersymmetric CP problem, since no extra CP-violating phases are generated.

\section{Dynamical Supersymmetry Breaking with $\langle F\rangle=0$ and $\langle W\rangle \neq 0$}

In supergravity theories the scalar potential is given by [4]

$$
V=K_{i \bar{\jmath}} F^{i} F^{\dagger \bar{\jmath}}-3 e^{K / M_{P}^{2}} \frac{|W|^{2}}{M_{P}^{2}}+\frac{1}{2} D_{a} D_{a},
$$

where $K$ is the Kähler potential, $K_{i \bar{\jmath}}$ is the Kähler metric, $W$ is the superpotential,

$$
F^{\dagger \bar{\jmath}}=-e^{K / 2 M_{P}^{2}} K^{i \bar{\jmath}}\left(\partial_{i} W+\partial_{i} K \frac{W}{M_{P}^{2}}\right),
$$

are the generalized $F$-terms of the chiral superfields and $D_{a}$ are the gauge $D$-terms of the vector superfields. We will consider models in which $\left\langle F_{i}\right\rangle=0$ but $\langle W\rangle \neq 0 \ll M_{P}^{3}$. The negative contribution to the cosmological constant coming from the second term of eq. (11) will be assumed to be cancelled out by a non-zero $D$-term. We will not specify how such a $D$-term is generated. We will rather take this fine tuning as an assumption. We will assume that the light states are neutral under this $D$-term, and therefore that this $D$-term does not play any role in the breaking of supersymmetry in the ordinary fields. In these models, it will be the non-zero gravitino mass $m_{3 / 2}=\left\langle e^{K / 2 M_{P}^{2}} W\right\rangle / M_{P}^{2}$ the source of low-energy supersymmetry breaking. 
A general (sufficient) condition for generating $\langle W\rangle \neq 0$ and vanishing $F$-terms can be easily formulated in supergravity theories: Any model of global supersymmetry with chiral superfield(s) $X$ that has a minimum that satisfies

(a) $\langle X\rangle=X_{\text {global }} \ll M_{P}$ and non-zero $\left\langle W\left(X_{\text {global }}\right)\right\rangle \ll M_{P}^{3}$,

(b) preserves global supersymmetry, $\left\langle\partial_{X} W\right\rangle=0$, and

(c) do not have chiral superfields with masses smaller than $\langle W\rangle / M_{P}^{2}$,

will have, in the locally supersymmetric extension, a local minimum at some $\langle X\rangle=X_{\text {grav }}$ with vanishing generalized $F$-terms and $\left\langle W\left(X_{\text {grav }}\right)\right\rangle \neq 0$.

The proof goes as follows. Due to (a) and (c) the gravity corrections to the global supersymmetric masses and VEVs are small. Therefore, these corrections cannot create a massless state, the would-be Goldstino, and supergravity cannot be broken, i.e., the $F$-terms must vanish.

Examples of dynamically generated superpotentials that do not break supersymmetry in the flat limit can be readily constructed. Models of this type are any strongly coupled SU(N) theory with a number of flavors less than $\mathrm{N}$ that are massive [5]. The simplest is $\mathrm{SU}(2)$ with one flavour (two doublets) $\psi, \bar{\psi}$. This theory has an unique $D$-flat direction that can be parametrized by a single holomorphic invariant ("meson") $X^{2}=\bar{\psi} \psi$. Far along the flat direction, the $\mathrm{SU}(2)$ is completely broken and the only low-energy (super)field is the massless $X$. By adding a mass term in the superpotential

$$
W=m X^{2},
$$

one can lift all the points of the vacuum but the origin $(X=0)$ in which the gauge symmetry is unbroken. Instantons, however, generate a superpotential which pushes $X$ away from the origin [6]:

$$
W=m X^{2}+\Lambda^{5} / X^{2}
$$

where $\Lambda$ is the strong scale of the $\mathrm{SU}(2)$. This stabilizes $X$ at

$$
\left\langle X^{2}\right\rangle=\sqrt{\frac{\Lambda}{m}} \Lambda^{2},
$$

and generates a non-zero vacuum expectation value (VEV) for the superpotential

$$
\langle W\rangle=2 \sqrt{\Lambda m} \Lambda^{2}
$$

without breaking supersymmetry. For $m \ll \Lambda, X$ can be treated as a canonically normalized superfield with mass $4 \mathrm{~m}$. Therefore, there are not massless states in the theory, and the conditions (a), (b) and (c) hold. It can be verified by explicit minimization that $\left\langle F_{X}\right\rangle$ is also zero in the supergravity case, in agreement with the general argument given above. By continuity this is also true for $m \gtrsim \Lambda$.

Another examples can be found in superpotentials generated from a quantum modified moduli space [5], e.g. [7]:

$$
W=\lambda X^{3}+\Lambda^{2} X
$$


where $\Lambda$ is the meson condensate of a strong $\mathrm{SU}(\mathrm{N})$ with a number of flavors equal to N. Also in these models we have vanishing $F$-terms and $\langle W\rangle \neq 0 \ll M_{P}^{3}$.

\section{Soft Supersymmetry Breaking Terms}

\subsection{Gravity-induced soft terms}

In supergravity theories the scalar soft masses are given by (for canonically normalized fields) [2]

$$
m_{i \bar{\jmath}}^{2}=-2 m_{3 / 2}^{2} \delta_{i \bar{\jmath}}+\frac{\left\langle F^{l}\right\rangle\left\langle F^{\dagger \bar{k}}\right\rangle}{M_{P}^{2}}\left[K_{l \bar{k}} \delta_{i \bar{\jmath}}-R_{l \bar{k} i \bar{\jmath}}\right],
$$

where $R_{l \bar{k} i \bar{\jmath}}$ is the curvature in the Kähler manifold. The last term in eq. (8) is responsible for the breaking of universality and inducing flavor violating interactions in the squark sector. One popular way to retain universality is to assume that the Kähler manifold is flat (minimal supergravity) [1]. Nevertheless, there is a priori no reason, based on symmetry principles, to expect that (from string theories one learns that universality can only arise under certain conditions [8]).

In theories with vanishing $\left\langle F_{i}\right\rangle$, universality is automatic since the last term of eq. (8) is zero. In these theories, we obtain

$$
m_{i \bar{\jmath}}^{2}=-2 m_{3 / 2}^{2} \delta_{i \bar{\jmath}} .
$$

The origin of universality can be understood as a consequence of the super-Weyl-Kähler symmetry

$$
\begin{aligned}
W & \rightarrow e^{-\mathcal{F} / M_{P}^{2}} W, \\
K & \rightarrow K+\mathcal{F}+\mathcal{F}^{\dagger},
\end{aligned}
$$

where $\mathcal{F}$ is an arbitrary holomorphic function of chiral superfields. To see that, let us write the effective supergravity Lagrangian in a flat gravitational background, using the superfield formalism [9, 10, 11]:

$$
\mathcal{L}=-3 M_{P}^{2} \int d^{4} \theta \varphi \varphi^{\dagger} e^{-K / 3 M_{P}^{2}}+\left(\int d^{2} \theta \varphi^{3} W+\text { h.c. }\right),
$$

where $\varphi$ is the compensator superfield; this is an auxiliary (non-dynamical) chiral superfield introduced in the theory to make the super-Weyl-Kähler symmetry manifest. For this purpose, $\varphi$ should transform as

$$
\varphi \rightarrow e^{\mathcal{F} / 3 M_{P}^{2}} \varphi
$$

under the super-Weyl-Kähler transformation. The compensator superfield can be eliminated by its equation of motion that gives [1]

$$
\varphi=e^{K / 6 M_{P}^{2}}\left[1+\theta^{2}\left(e^{K / 2 M_{P}^{2}} \frac{W^{\dagger}}{M_{P}^{2}}+\frac{\partial_{i} K F^{i}}{3 M_{P}^{2}}\right)\right] .
$$


For $\langle W\rangle \neq 0, \varphi$ breaks supersymmetry. The super-Weyl-Kähler symmetry guarantee that $\varphi$ couples universally to matter and therefore that the scalar fields receive universal soft masses. The result of eq. (9) can be easily obtained by inserting eqs. (2) and (13) in eq. (11).

Trilinears and bilinears soft terms can be also obtained by the same procedure. One obtains

$$
\begin{aligned}
& A_{i j k}=0, \\
& B_{i j}=-m_{3 / 2} M_{i j},
\end{aligned}
$$

where $M_{i j}$ are the bilinear couplings in the superpotential. Since the trilinears vanish, there are not left-right flavor-violating interactions in these theories. This is because the super-Weyl-Kähler symmetry does not allowed for a coupling between $\varphi$ and the vector superfield which transforms as a singlet under this symmetry. Nevertheless, as we will see in the next section, gaugino masses can arise at the one-loop level.

Let us finally comment on the supersymmetric mass of the Higgs, $\int d^{2} \theta \mu H_{u} H_{d}$. If this term is not allowed in the superpotential (by an $R$-symmetry), but it is present in the Kähler, $\lambda H_{u} H_{d} \in K$, the first term of eq. (11) will generate the coupling

$$
\int d^{4} \theta \varphi \varphi^{\dagger}\left(\lambda H_{u} H_{d}+\text { h.c. }\right) \text {. }
$$

After supersymmetry breaking, we obtain by replacing eq. (13) in eq. (16)

$$
\int d^{2} \theta \mu H_{u} H_{d}
$$

where $\mu=\lambda m_{3 / 2}$ is of the right order of magnitude. This is just the mechanism of Ref. [13]. Eq. (16) also generates a bilinear soft term for the Higgs, $B \mu=-\lambda m_{3 / 2}^{2}$.

\subsection{Low-energy soft masses}

Although the gaugino masses are zero at $M_{P}$, they can be generated at the one-loop level if there are heavy chiral superfields in the theory [14. Each heavy superfield gives a contribution to the gaugino masses at the one-loop level:

$$
m_{\lambda_{a}}=-\frac{\alpha_{a}}{4 \pi} \sum_{R} S_{a}(R) m_{3 / 2}
$$

where $S_{a}(R)$ is the Dynkin index of a heavy superfield in the $R$ representation of the gauge group $G_{a}\left(S_{a}=1 / 2\right.$ for the fundamental representation). In GUTs the one-loop contribution of eq. (18) can be quite large due to the large number of heavy fields. One can expect $m_{\lambda_{a}} \sim$ $m_{3 / 2}$. In GUTs there are also contributions from the heavy vector multiplets [14]. These contributions do not respect the GUT symmetry, and therefore provide deviations from gaugino mass unification.

\footnotetext{
${ }^{1}$ It is interesting to mention that these interactions are usually present even in theories with non-Abelian flavor symmetries and can lead to large dipole moment transitions [12].
} 
In the superfield formalism of eq. (11), the gaugino masses of eq. (18) can be understood as arising from the heavy-field contribution to the super-Weyl-Kähler anomaly [10]:

$$
\frac{\sum_{R} S_{a}(R)}{32 \pi^{2}} \int d^{2} \theta \ln \varphi W_{a} W_{a}
$$

where $W_{a}$ is the superfield strength of the gauge vectors. Inserting eq. (13) in the above equation, we recover eq. (18). Eq. (18) holds at the scale at which the heavy fields are integrated out; to obtain the values of the gaugino masses at a low-energy scale, one still has to evolve them down using the RGE of the supersymmetric standard model.

The scalar soft masses also receive radiative corrections from the low-energy spectrum. The degeneracy of the first and second family squarks will not be altered if their Yukawa couplings remain small below $M_{P}$. In theories with no extra $\mathrm{U}(1)$, the scalar masses at the weak scale will be given by (neglecting the contribution coming from the $\mathrm{SU}(2)_{L} \times \mathrm{U}(1)_{Y} D$-term and Yukawa couplings

$$
m_{i}^{2}=-2 m_{3 / 2}^{2}+a_{i} m_{\lambda}^{2}\left(M_{G}\right)
$$

where $a_{i} \sim 5-7$ for the squarks, $\sim 0.5$ for the left-handed sleptons and $\sim 0.15$ for the righthanded sleptons; $m_{\lambda}\left(M_{G}\right)$ is the one-loop gaugino mass induced by the heavy fields that we assumed to be at the GUT scale. The requirement of non-tachionic selectrons, i.e., $m_{\tilde{e}_{L, R}}^{2}>0$, puts a strong constraint on the gaugino mass, $m_{\lambda}\left(M_{G}\right) \gtrsim 3.6 m_{3 / 2}$. Since the gaugino mass arises at one-loop, this constraint is difficult to satisfy. Extra contributions to the scalar masses can arise if extra $\mathrm{U}(1)$ are present in the theory as we will consider in the next section.

\subsection{Models with an extra $\mathrm{U}(1)$}

A very well motivated candidate for a gauge $\mathrm{U}(1)$-symmetry is the anomalous $\mathrm{U}(1)$ often present in string theories. The anomaly is cancelled by the Green-Schwartz mechanism which requires non-zero (and equal) mixed anomalies for each gauge group [15]. A non-zero gravitational anomaly results into the appearance of a Fayet-Iliopoulos (FI) term $\xi=\mathcal{O}\left(M_{P}^{2} \operatorname{Tr} \mathbf{Q}\right)$. In string theories the FI-term can be calculated and is given by [16] $\xi=g^{2} \operatorname{Tr} \mathbf{Q} M_{P}^{2} /\left(192 \pi^{2}\right)$. We will assume that $\operatorname{Tr} \mathbf{Q}<0$. Below $M_{P}$, the potential is given by

$$
V=-2 m_{3 / 2}^{2} \sum_{i}\left|\phi_{i}\right|^{2}+\left|\frac{\partial W}{\partial \phi_{i}}\right|^{2}+\frac{g^{2}}{2}\left(\sum_{i} q_{i}\left|\phi_{i}\right|^{2}+\xi\right)^{2}+\mathcal{O}\left(m_{3 / 2}^{4}\right) .
$$

From the minimization of (21), one can see that, among the fields with $F$-flat directions, the one with the smallest (positive) charge $q$ will get a VEV

$$
\left\langle|\phi|^{2}\right\rangle=\frac{1}{q}\left(\frac{2 m_{3 / 2}^{2}}{g^{2} q}-\xi\right)
$$

\footnotetext{
${ }^{2}$ For the scalar top and Higgs, the effect of the Yukawa couplings should be included. A complete analysis of these masses and the electroweak breaking is beyond the scope of this letter.
} 
and a non-zero $D$-term will be generated ${ }^{\text {f }},\langle D\rangle=2 m_{3 / 2}^{2} /(q g)$. The non-zero $D$-term contributes to the soft masses of all the charged scalars [17, 18 f and we have at the scale $\sqrt{\xi}$

$$
m_{i}^{2}=2\left(\frac{q_{i}}{q}-1\right) m_{3 / 2}^{2} .
$$

Thus, fields with $q_{i}>q$ will have positive scalar soft masses. We will require that all matter fields as well as any field corresponding to a (exactly) flat direction satisfy this requirement and, therefore, there are not tachionic states (alternative scenarios could also be possible). The simplest model along these lines has the following content: (1) The superfield $\phi$. (2) The quark and lepton superfields with universal U(1)-charge $q_{Q}$ larger than $q$. (3) Two Higgs doublets $H_{u}$ and $H_{d}$; the charge of $H_{u}$ is taken to be $-2 q_{Q}$ to allow for a top-quark mass being generated from a renormalizable interaction $H_{u} Q \bar{U}$ (the charge of $H_{d}$ is more model dependent). (4) Extra (heavy) superfields $\Psi$ and $\bar{\Psi}$ vector-like under the standard model gauge group. The motivation for these states is two-fold. First, they are required for the mixed anomalies, and secondly they will contribute to the gaugino masses in accordance with the discussion in the previous section. They will get masses from their coupling to $\phi$ :

$$
\frac{\phi^{n}}{M_{P}^{n-1}} \Psi \bar{\Psi}, \quad n=-\frac{q_{\Psi}+q_{\bar{\Psi}}}{q} .
$$

In these models the $\mu$-parameter can also be generated from a higher dimensional coupling of $H_{u} H_{d}$ to $\phi$. For example, if $H_{u}$ and $H_{d}$ carry equal charges (in this case the bottom mass is also generated from a renormalizable interaction), the supersymmetric term

$$
\frac{\phi^{n}}{M_{P}^{n-1}} H_{u} H_{d}, \quad n=\frac{4 q_{Q}}{q},
$$

gives rise to a realistic value of $\mu$ for $\sqrt{\xi} / M_{P} \sim 0.1-0.01$ and $q_{Q} \sim(2-3) q$. Alternatively, the $\mu$-term could be generated from a coupling of $H_{u} H_{d}$ to a neutral singlet $S$ [19, 14]:

$$
W=S H_{u} H_{d}+S^{3} .
$$

(The cubic term is necessary for eliminating an unwanted weak-scale axion). Since $S$ is neutral under the anomalous U(1), its soft mass is negative. This drives a VEV for $S$ that generates the $\mu$-parameter.

Similar scenario could arise with non-anomalous U(1). An anomaly-free U(1) is easy to obtain, since the $\Psi-\bar{\Psi}$ states and matter states give opposite contributions to the anomalies. In this case, a FI-term of order $\xi \sim m_{3 / 2} M_{P}$ can be generated at the one-loop level if there is a mixing between the $\mathrm{U}(1) D$-term and the $D$-term that cancels the cosmological constant. This occurs whenever $\operatorname{Tr} \mathbf{Q} \mathbf{Q}^{\prime} \neq 0$ where $\mathbf{Q}$ and $\mathbf{Q}^{\prime}$ are the generators associated to the two $D$-terms.

\footnotetext{
${ }^{3}$ An $F$-term for $\phi$ is also generated in this model, $F_{\phi}=m_{3 / 2}\langle\phi\rangle$, but its contribution to the soft masses is small since $\langle\phi\rangle \ll M_{P}$.

4 This contribution can overwhelm the gravity-induced contribution if the anomalous U(1) is responsible for supersymmetry breaking [18]. This is an alternative way to suppress flavor-violating interactions.

${ }^{5}$ We could also consider a flavor-dependent charge assignment. The only crucial condition, to be consistent with flavor-violating constraints, is to equally charge the first and second family of down-quarks.
} 


\section{Phenomenological Implications}

The most important implication of the models with vanishing $F$-terms is that they lead to a mass degeneracy between the first and second family of squarks, and avoid dangerous flavorviolating interactions. It is also important to remark that these scenarios allow for gauging flavor symmetries $G_{f}$ without inducing a VEV for the $D$-term of $G_{f}$ (these $D$-terms are very dangerous since they usually break the interfamily degeneracy). A non-zero $D$-term is avoided if the fields that break $G_{f}$ have universal soft masses; this can be the case in our scenarios.

Another implication of the models is that the soft terms depend only on one parameter, the gravitino mass $m_{3 / 2}$. This allows for a solution to the supersymmetric CP problem [3]. The origin of this problem resides in the phases of the soft terms and $\mu$-parameter that induce, at the one-loop level, too large electric dipole moments. Even the minimal supergravity model suffers from this problem. In our models, however, all the phases can be rotated away. If $\mu$ arises from a supersymmetric term, like in eq. (17), (25) or (26), its phase can be eliminated by a rotation of the Higgs superfields. In the soft terms the only possible phase appears in $m_{3 / 2}$ that can be eliminated by an $R$-rotation.

The model of section 3.3 also allows for a solution to the strong CP problem. If $\mu$ is induced from eq. (25), there is a remnant Peccei-Quinn symmetry broken by the VEV of $\phi$. The axion is the superposition of the imaginary part of $\phi$ and the dilaton, orthogonal to the one eaten up by the gauge $\mathrm{U}(1)$ field.

A final important feature of models with vanishing $F$-terms is that they do not suffer from the Polonyi problem [20. This problem arises in conventional supergravity theories because supersymmetry is broken by non-vanishing $F$-terms of the hidden sector fields. In our case, $F$-terms vanish and the Polonyi problem does not arise (the only field that gets a non-zero $F$-term is $\varphi$ that is not a dynamical field).

\section{Conclusions}

We have considered a class of supergravity theories in which the hidden superfields have vanishing F-terms but a non-zero superpotential. This is generated dynamically at a low-energy and is the source of supersymmetry breaking in the observable sector as soon as the cosmological constant is set to zero by a non-zero $D$-term.

The gravity-induced soft supersymmetry breaking terms depend only on the gravitino mass and not on the details of the hidden sector. We find

$$
\begin{aligned}
& m_{i \bar{\jmath}}^{2}=-2 m_{3 / 2}^{2} \delta_{i \bar{\jmath}}, \\
& A_{i j k}=0, \\
& B_{i j}=-m_{3 / 2} M_{i j},
\end{aligned}
$$

for the scalar masses, trilinear and bilinear soft terms respectively. This universal pattern of 
soft masses guarantees the absence of flavor-violating interactions. No new CP-violating phases appear. The mechanism of ref. [13] to generate a $\mu$-parameter is also operative in these theories.

The negative soft masses in eq. (27) can become positive at low-energies if extra U(1) are present in the theory. We have considered models in which the matter fields transform under an anomalous $\mathrm{U}(1)$. This is often the case in string theories. In such cases, the soft masses receive a positive contribution (see eq. (23)). The gaugino masses are zero at $M_{P}$, but they are induced at the one-loop level

$$
m_{\lambda_{a}}=-\frac{\alpha_{a}}{4 \pi} \sum_{R} S_{a}(R) m_{3 / 2}
$$

if heavy states between $M_{P}$ and the weak scale, transforming under the standard model group, are present in the theory. Thus, the gaugino masses carry us information about the GUT physics.

Unlike conventional supergravity models, there are not cosmological problems in the hidden sector since there are not Polonyi-like states with large VEVs and small masses.

It is a pleasure to thank R. Barbieri, E. Dudas, R. Rattazzi and C. Savoy for very useful discussions. 


\section{References}

[1] R. Barbieri, S. Ferrara and C.A. Savoy, Phys. Lett. B119 (1982) 343; A.H. Chamseddine, R. Arnowitt and P. Nath, Phys. Rev. D49 (1982) 970.

[2] S.K. Soni and H.A. Weldon, Phys. Lett. B119 (1982) 343.

[3] W. Buchmüller and D. Wyler, Phys. Lett. B121 (1983) 321; J. Polchinski and M.B. Wise, Phys. Lett. B125 (1983) 393.

[4] E. Cremmer et al., Phys. Lett. B79 (1978) 231; Nucl. Phys. B147 (1979) 105; E. Witten and J. Bagger, Phys. Lett. B115 (1982) 202; E. Cremmer,S. Ferrara, L. Girardello and A. van Proeyen, Phys. Lett. B116 (1982) 231; Nucl. Phys. B212 (1983) 413; J. Bagger, Nucl. Phys. B211 (1983) 302.

[5] For a review see, K. Intriligator and N. Seiberg, hep-th/9509066, and references therein.

[6] I. Affleck, M. Dine and N. Seiberg, Nucl. Phys. B256 (1985) 557; A.I. Vainshtein, V.I. Zakharov and M.A. Shifman, Sov. Phys. Usp. 28 (1985) 709; D. Amati, K. Konishi, Y. Meurice, G. Rossi and G. Veneziano Phys. Rep. 163 (1988) 169.

[7] M. Shifman, hep-th/9704114; S. Dimopoulos, G. Dvali and R. Rattazzi, hep-ph/9705348.

[8] L. Ibañez and D. Lust, Nucl. Phys. B382 (1992) 305; V. Kaplunovsky and J. Louis, Phys. Lett. B306 (1993) 269; A. Brignole, L. Ibañez and C. Muñoz, Nucl. Phys. B422 (1994) 125.

[9] T. Kugo and S. Uehara, Nucl. Phys. B222 (1983) 125.

[10] V. Kaplunovsky and J. Louis, Nucl. Phys. B422 (1994) 57.

[11] J. Bagger, E. Poppitz and L. Randall, Nucl. Phys. B455 (1995) 59.

[12] See, for example, A. Pomarol and D. Tommasini, Nucl. Phys. B466 (1996) 3; R. Barbieri, G. Dvali and L.J. Hall, Phys. Lett. B377 (1996) 76.

[13] G.F. Giudice and A. Masiero, Phys. Lett. B206 (1988) 480.

[14] J.P. Derendinger and C.A. Savoy, Nucl. Phys. B237 (1984) 307.

[15] M. Green and J. Schwarz, Phys. Lett. B149 (1984) 117.

[16] J. Atick, L. Dixon and A. Sen, Nucl. Phys. B292 (1987) 109; M. Dine, I. Ichinose and N. Seiberg, Nucl. Phys. B293 (1987) 253.

[17] H. Nakano, hep-th/9404033; E. Dudas, S. Pokorski and C.A. Savoy, Phys. Lett. B369 (1996) 255; Y. Kawamura and T. Kobayashi, Phys. Lett. B375 (1996) 141; E. Dudas, C. Grojean, S. Pokorski and C.A. Savoy, Nucl. Phys. B481 (1996) 85. 
[18] G. Dvali and A. Pomarol, Phys. Rev. Lett. 77 (1996) 3728; P. Binetruy and E. Dudas, Phys. Lett. B389 (1996) 503.

[19] H.P. Nilles, M. Srednicki and D. Wyler, Phys. Lett. B120 (1983) 346.

[20] G.D. Coughlan, W. Fischler, E.W. Kolb, S. Raby and G.G. Ross, Phys. Lett. B131 (1983) 59; J. Ellis, D.V. Nanopoulos and M. Quirós, Phys. Lett. B147 (1986) 176. 